\title{
Restoration of a whole-sky CMB map
}

\author{
Jaiseung Kim ${ }^{* a b}$, Pavel Naselsky ${ }^{b c}$, Nazzareno Mandolesi ${ }^{d}$ \\ ${ }^{a}$ Max Planck Institut für Astrophysik, Karl-Schwarzschild-Str. 1, 85748 Garching, Germany \\ ${ }^{b}$ Niels Bohr Institute, Blegdamsvej 17, DK-2100 Copenhagen, Denmark \\ ${ }^{c}$ Discovery Center, Blegdamsvej 17, DK-2100 Copenhagen, Denmark \\ ${ }^{d}$ INAF/IASF-BO, Istituto di Astrofisica Spaziale e Fisica Cosmica di Bologna \\ via Gobetti 101, I-40129 Bologna, Italy \\ E-mail: Kimempa-qarching.mpq.de, haselskyenbi.dr, \\ mandolesidiasfbo.inaf.it
}

Several astrophysical emission sources (the "foreground") exist between the last scattering surface and our vantage point. Due to the foreground contamination, certain area of CMB data is masked out, leading to large cuts around the Galactic equator and numerous holes. Since many CMB analyses, may be performed on a whole sky map in a straightforward and more reliable way, it is of utmost importance to develop an efficient method to restore a whole-sky CMB map from incomplete sky data, while preserving statistical properties. Though there is an well-established method to produced Gaussian random field under constraints, we may not readily implement the method on the pixellized CMB data, due to prohibitive computational cost. Noting this, we considered implementing the method in harmonic space, where the computational cost is significantly reduced with good approximation. We validated our implementation with simulations, and confirm restoration is made in a way compliant with the expected statistical properties. Subsequently, we restored a whole-sky map from the masked WMAP sky data, and investigated a known CMB data anomaly of lowest multipoles. Our method will be complementary to other efforts on restoring or reconstructing the masked CMB data, and of great use for Planck surveyor and future missions.

Big Bang, Big Data, Big Computers,

September 19-21, 2012

Laboratoire Astroparticule et Cosmologie, 10 rue A. Domon et L. Duquet, 75205 Paris 13, France

*Speaker. 


\section{Contents}

[1. Introduction []

2. CMB anisotropy in harmonic space []

3. In-painting in harmonic space

4. Application to simulated data

5. Application to the WMAP data

6. Discussion 田

\section{Introduction}

There exist several astrophysical emission sources between the last scatteringfor surface and our vantage point. Due to the contamination from the 'foregrounds', we need to apply proper masking on microwave sky maps, which leads to cut of varying width around the Galactic equator and numerous holes. Since many CMB analysis, in particular on the largest angular scales, may be performed on a whole-sky map in a straightforward and more reliable manner, there have been many efforts to reconstruct a whole-sky map from incomplete sky data [四-团]. However, the fidelity of reconstruction is limited, because it is not possible to reliably reconstruct harmonics modes mainly confined to the Galactic cuts [ [ $]$ ]. Therefore, there have been active attempts to fill in the missing information in CMB sky data with a priori [[]-[].

Historically, the act of recovering damaged parts of valuable paintings by a skilled restoration artist is called 'in-painting'. In digital imaging, there are various in-painting methods [ [ $\mathrm{B}-\mathrm{W}]$. While these methods work well for images of periodic or predictable patterns, they may not be suitable for CMB data, which have random Gaussian nature. On the other hand, there have been works on generating constrained Gaussian fields, which have been used in the study of largescale structures [12- [4]]. However, it is not feasible for the pixel data of the WMAP or Planck surveyor, which amounts to millions of pixels or more. Noting this, we consider implementing the method in harmonic space, where the computational load may be significantly reduced with good approximation. After validating it with simulation, we are going to apply the method to the mask WMAP foreground-reduced maps and investigate the well-known anomaly of lowest multipoles. Throughout this work, we will use the term 'in-painting' and 'restoring' interchangeably. 


\section{CMB anisotropy in harmonic space}

CMB anisotropy over a whole-sky is conveniently decomposed in terms of spherical harmonics:

$$
T(\hat{\mathbf{n}})=\sum_{l m} a_{l m} Y_{l m}(\hat{\mathbf{n}})
$$

where $a_{l m}$ and $Y_{l m}(\theta, \phi)$ are a decomposition coefficient and a spherical harmonic function, and $\hat{\mathbf{n}}$ denotes a sky direction. In the standard cosmological model, decomposition coefficients of CMB anisotropy follow the Gaussian distribution of the following statistical properties:

$$
\begin{aligned}
\left\langle a_{l m}\right\rangle & =0, \\
\left\langle a_{l m} a_{l^{\prime} m^{\prime}}^{*}\right\rangle & =\delta_{l l^{\prime}} \delta_{m m^{\prime}} C_{l},
\end{aligned}
$$

where $\langle\ldots\rangle$ denotes the average over an ensemble of universes, and $C_{l}$ denotes CMB power spectrum, and $\delta_{l l^{\prime}}$ and $\delta_{m m^{\prime}}$ are Kronecker Delta functions. Accordingly, the CMB anisotropy $T(\theta, \phi)$, which follows Gaussian distribution, have the following angular correlation:

$$
\left\langle T(\hat{\mathbf{n}}) T\left(\hat{\mathbf{n}}^{\prime}\right)\right\rangle=\sum_{l} \frac{2 l+1}{4 \pi} W_{l} C_{l} P_{l}(\cos \theta),
$$

where $W_{l}$ is a beam smoothing function of the observation, $P_{l}$ is a Legendre polynomials and $\theta=\cos ^{-1}\left(\hat{\mathbf{n}} \cdot \hat{\mathbf{n}}^{\prime}\right)$.

In the presence of a foreground mask, the spherical coefficients of a masked sky $\tilde{a}_{l m}$ are related to those of a whole-sky as follows:

$$
\tilde{a}_{l_{3} m_{3}}=\sum_{l_{2} m_{2}} F\left(l_{2}, m_{2}, l_{3}, m_{3}\right) a_{l_{2} m_{2}}
$$

where

$$
\begin{gathered}
F\left(l_{2}, m_{2}, l_{3}, m_{3}\right) \\
=(-1)_{3}^{m} \sqrt{\frac{2 l_{3}+1}{4 \pi}} \sum_{l_{1} m_{1}} \sqrt{\left(2 l_{1}+1\right)\left(2 l_{2}+1\right)}\left(\begin{array}{ccc}
l_{1} & l_{2} & l_{3} \\
m_{1} & m_{2} & -m_{3}
\end{array}\right)\left(\begin{array}{ccc}
l_{1} & l_{2} & l_{3} \\
0 & 0 & 0
\end{array}\right) w_{l_{1} m_{2}}, \\
w_{l_{1} m_{2}}=\int Y_{l_{1} m_{1}}^{*}(\theta, \phi) W(\theta, \phi) d \Omega,
\end{gathered}
$$

and $W(\theta, \phi)$ is the mask function, which is zero inside the mask and one elsewhere, and the terms with the big parenthesis being Wigner 3j symbols. Using Eq. 2.3 and 2.5 , we may show that there exist the following relation:

$$
\begin{gathered}
\left\langle a_{l m} \tilde{a}_{l^{\prime} m^{\prime}}^{*}\right\rangle=F^{*}\left(l, m, l^{\prime}, m^{\prime}\right) C_{l}, \\
\left\langle\tilde{a}_{l^{\prime} m^{\prime}} \tilde{a}_{l^{\prime \prime} m^{\prime \prime}}^{*}\right\rangle=\sum_{l m} F^{*}\left(l, m, l^{\prime}, m^{\prime}\right) F\left(l, m, l^{\prime \prime}, m^{\prime \prime}\right) C_{l} .
\end{gathered}
$$

Unlike the whole-sky case (c.f. Eq. [2.3]), there are non-zero off-diagonal correlation in the masked sky. 


\section{In-painting in harmonic space}

$2 *$ nside There exists an efficient algorithm on the simulation of constrained Gaussian fields developed by [14]. According to the work, we may simulate Gaussian random field $f(\mathbf{r})$ under constraints $f\left(\mathbf{r}_{j}\right)$ by the following:

$$
f(\mathbf{r})=f_{\mathrm{mc}}(\mathbf{r})+\sum_{i j} \mathbf{b}_{i}\left(\mathbf{C}^{-1}\right)_{i j}\left(f\left(\mathbf{r}_{j}\right)-f_{\mathrm{mc}}\left(\mathbf{r}_{j}\right)\right),
$$

where

$$
\begin{aligned}
\mathbf{b}_{i} & =\left\langle f(\mathbf{r}) f\left(\mathbf{r}_{i}\right)\right\rangle, \\
\mathbf{C}_{i j} & =\left\langle f\left(\mathbf{r}_{i}\right) f\left(\mathbf{r}_{j}\right)\right\rangle,
\end{aligned}
$$

and the subscript 'mc' denotes unconstrained Gaussian Monte-Carlo simulation. Applying Eq. [.] to CMB pixel data, we may restore the values of the masked pixels [U]. Though in a slight different context, the power spectrum estimation by the Gibbs sampling, which includes generating an underlying CMB map according to the conditional distribution, have some overlapping [155, [6]].

As seen in Eq. 2.4 and Fig. [1], there exist pixel correlation at wide range of separation angles, which makes us to take into account tremendous amount of pixels even for in-painting a single pixel. Therefore, we may not readily apply the method to the modern data, which have millions of pixels. In the limit of a whole-sky coverage and a homogeneous noise, the covariance in harmonic space becomes diagonal (c.f. [2.3). Therefore, harmonic space may provide the optimal environment than the pixel space, as far as the unmasked area is much greater than the masked area. Let us consider in-painting in harmonic space. Since CMB anisotropy in harmonic space (i.e. $a_{l m}$ ) are expected to follow Gaussian distribution, we may simply rewrite Eq. B.] for the CMB anisotropy in harmonic space as follows:

$$
a_{l m}=a_{l m}^{\mathrm{mc}}+\mathbf{b} \mathbf{C}^{-1}\left(\tilde{\boldsymbol{a}}-\tilde{\boldsymbol{a}}^{\mathrm{mc}}\right),
$$

where $\tilde{\mathbf{a}}$ is a column vector consisting of $\tilde{a}_{l^{\prime} m^{\prime}}$ and

$$
\begin{aligned}
\mathbf{b} & =\left\langle a_{l m} \tilde{\boldsymbol{a}}^{\dagger}\right\rangle, \\
\mathbf{C} & =\left\langle\tilde{\boldsymbol{a}} \tilde{\boldsymbol{a}}^{\dagger}\right\rangle .
\end{aligned}
$$

As discussed previously, the off-diagonal elements of the covariance matrix $\mathbf{C}$ are non-zero, which may require a inversion of a huge matrix. However, let us consider the widely foreground mask, which is shown in Fig. $\mathbb{W}$. In the same figure, we show $W_{l}=(2 l+1)^{-1} \sum_{m}\left|w_{l m}\right|^{2}$, which indicates the multipole dependence of the mask in harmonic space. As shown in Fig. 四, the magnitude of $w_{l m}$ are only significant at lowest multipoles (i.e. $w_{l m} \approx 0(l \gg 1)$ ). Using this and Eq. 2.6 and the triangular inequalities of Wigner $3 \mathrm{j}$ symbol, we may see $F^{*}\left(l, m, l^{\prime}, m^{\prime}\right) \approx 0$ for $\left|l-l^{\prime}\right| \gg 1$. This indicates $a_{l m}$ are almost independent of $\tilde{a}_{l^{\prime} m^{\prime}}$ for $\left|l-l^{\prime}\right| \gg 1$ (c.f. Eq. [2.8). Therefore, we need to consider only constraints $\tilde{a}_{l^{\prime} m^{\prime}}$, whose multipoles do not differ from those of the interested $a_{l m}$ very much. Given this approximation, we may implement in-painting by Eq. [.2.], 

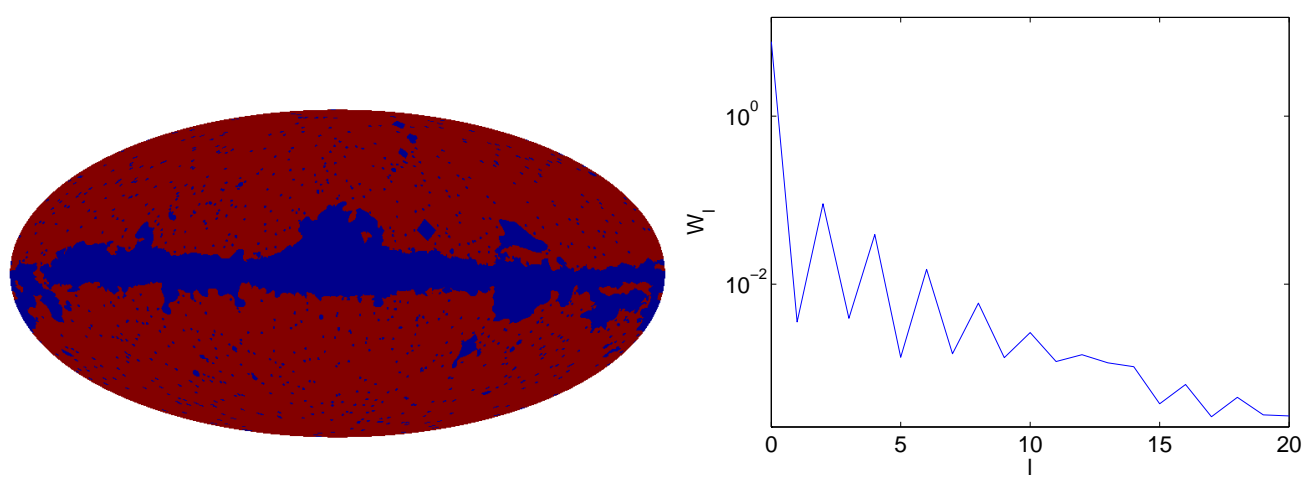

Figure 1: The WMAP teams' KQ85 foreground mask (left), $W_{l}=(2 l+1)^{-1} \sum_{m}\left|w_{l m}\right|^{2}$ (right)

without incurring prohibitive computational cost and losing much accuracy. We may summarize the procedure as follows: First, we generate unconstrained $a_{l m}$ by Monte-Carlo simulation, and then transform them by Eq. 3.2. From the result of Eq. B.2, we synthesize CMB anisotropy map $T(\theta, \phi)$, as necessary.

\section{Application to simulated data}

In order to validate our method, we first generated simulated CMB data, where we assumed the WMAP concordance $\Lambda$ CDM model and the WMAP beam smoothing at V band [प], प8]. We set the simulation to contain the multipoles up to 700, and produced it with the HEALPix pixellization Nside $=512$. We masked the simulated data by the WMAP KQ85, which admits pixel data of sky fractions $78 \%$. At the top of Fig. [, we show the simulated map with the foreground mask applied.
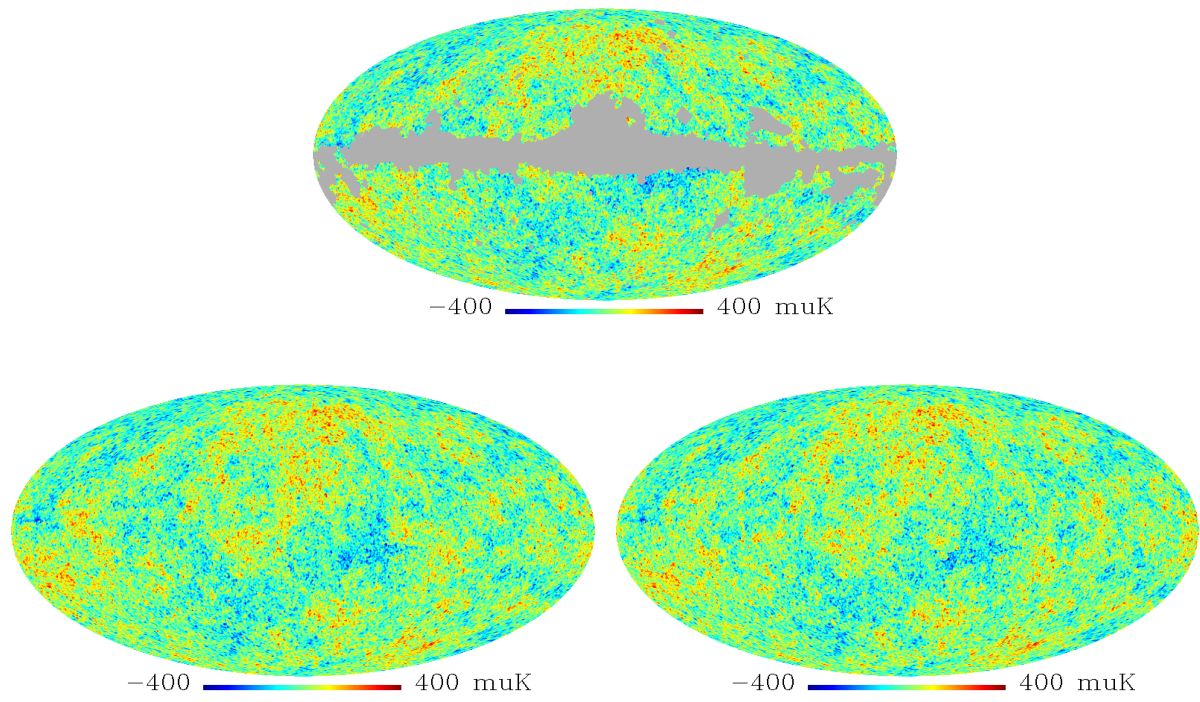

Figure 2: the simulated input map with the KQ85 mask applied (top), restored maps (middle, bottom): both are equally likely, given the constraints 
We applied the procedures described in the previous section to the masked simulated data, and restored a whole-sky map from the masked map. In Fig. ఐ, we show two restored maps, which are obtained from different unconstrained realizations (i.e. the terms of subscript 'mc' in Eq. B.2). Both of them are equally likely, given the constraints. Given the random Gaussian nature of CMB

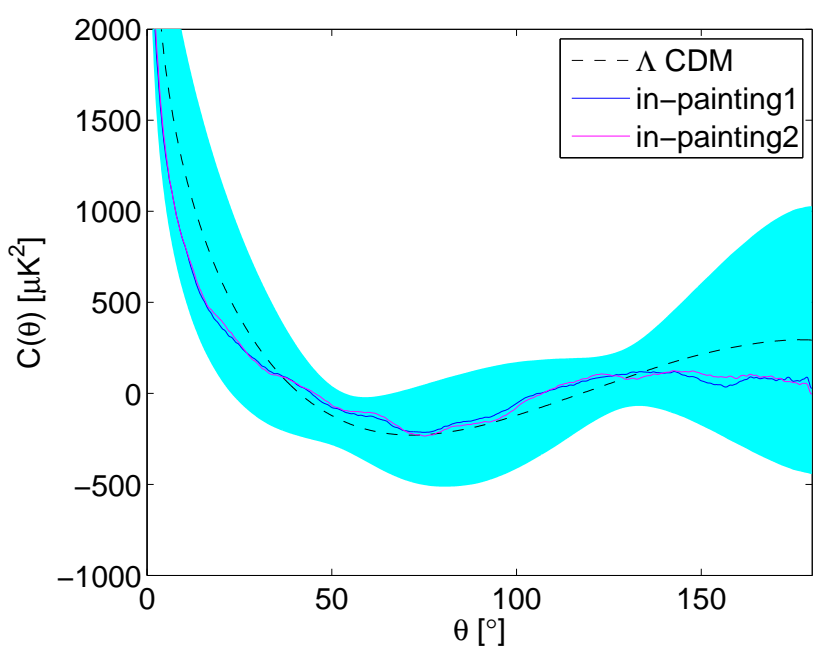

Figure 3: angular autocorrelation of restored maps

anisotropy, it is not possible to reconstruct the exact realization, which happens to be our Universe. Therefore, our intention is filling-in the missing information in a way compliant with the expected statistical properties. In order to see that our restored maps, indeed, satisfy the expected statistical properties, we estimated angular correlations, which are plotted in Fig. 2.4. In the same plot, we show the angular correlation of the WMAP concordance model [ㅍ] ], where the dotted line and shaded region denote the theoretical prediction and $2 \sigma$ ranges. As shown in Fig. Bl, the angular correlation of our restored maps are in good agreement with the theoretical expectation.

\section{Application to the WMAP data}

In order to reduce foreground contamination in microwave sky data, the WMAP team subtracted diffuse foregrounds by template fitting, and produced 'foreground-reduced maps', which are available at $\mathrm{Q}, \mathrm{V}$ and $\mathrm{W}$ band respectively [ㅁ]. Besides the foreground-reduced maps, there is the Internal Linear Combination (ILC) map, which is often used without foreground masking. Despite contamination from bright point sources and Galactic foregrounds in the ILC map, the whole-sky ILC map is widely used for study of non-Gaussianity, because of difficulty of investigating lowest multipoles on a masked sky data [20-[36]. Not surprisingly, foreground-reduced maps with foreground mask is more reliable a36nd contains less foreground contamination than the whole-sky ILC map. Therefore, it is worth to investigate the anomaly at lowest multipoles, using the foreground-reduced maps. By using our method, we restored a whole-sky map from masked foreground-reduced maps, where the WMAP team's KQ85 mask is used. The restoration process is the same with the one described in the previous sections, except that $C_{l}$ in Eq. 2.9 should be replaced by $C_{l}+N_{l}$ with $N_{l}$ being the power spectrum of instrument noise. In order to estimate 

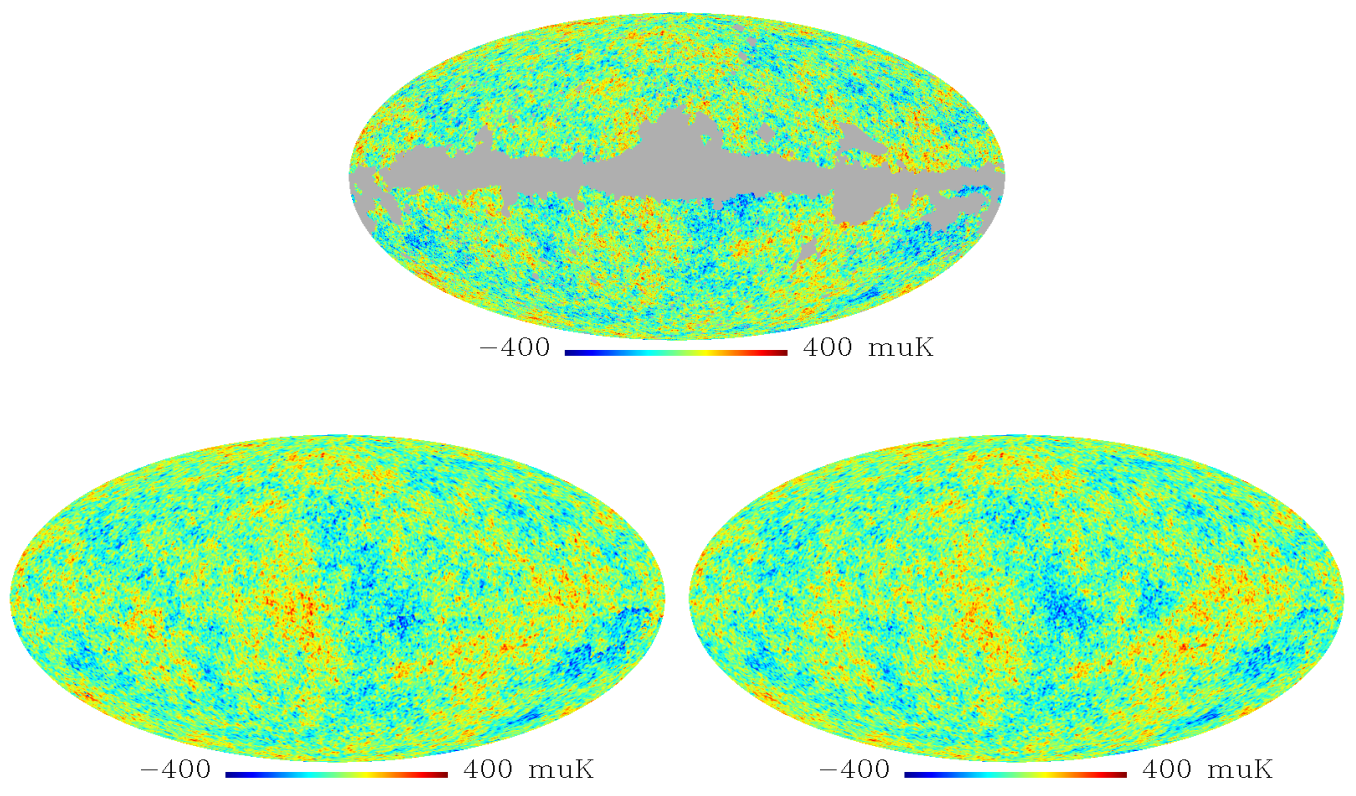

Figure 4: Restored maps from the foreground-reduced map of Q band, two restored maps (middle and bottom)
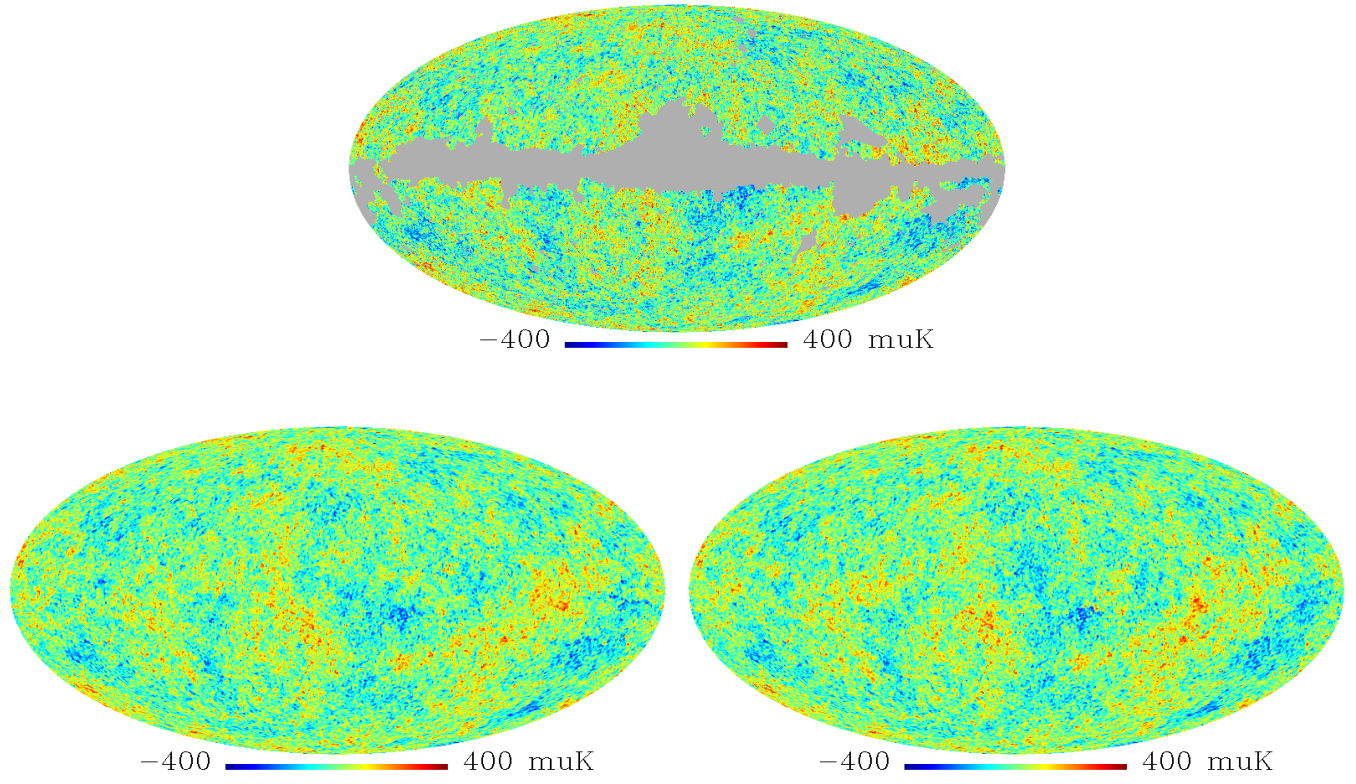

Figure 5: Restored maps from the foreground-reduced map of V band, two restored maps (middle and bottom) 

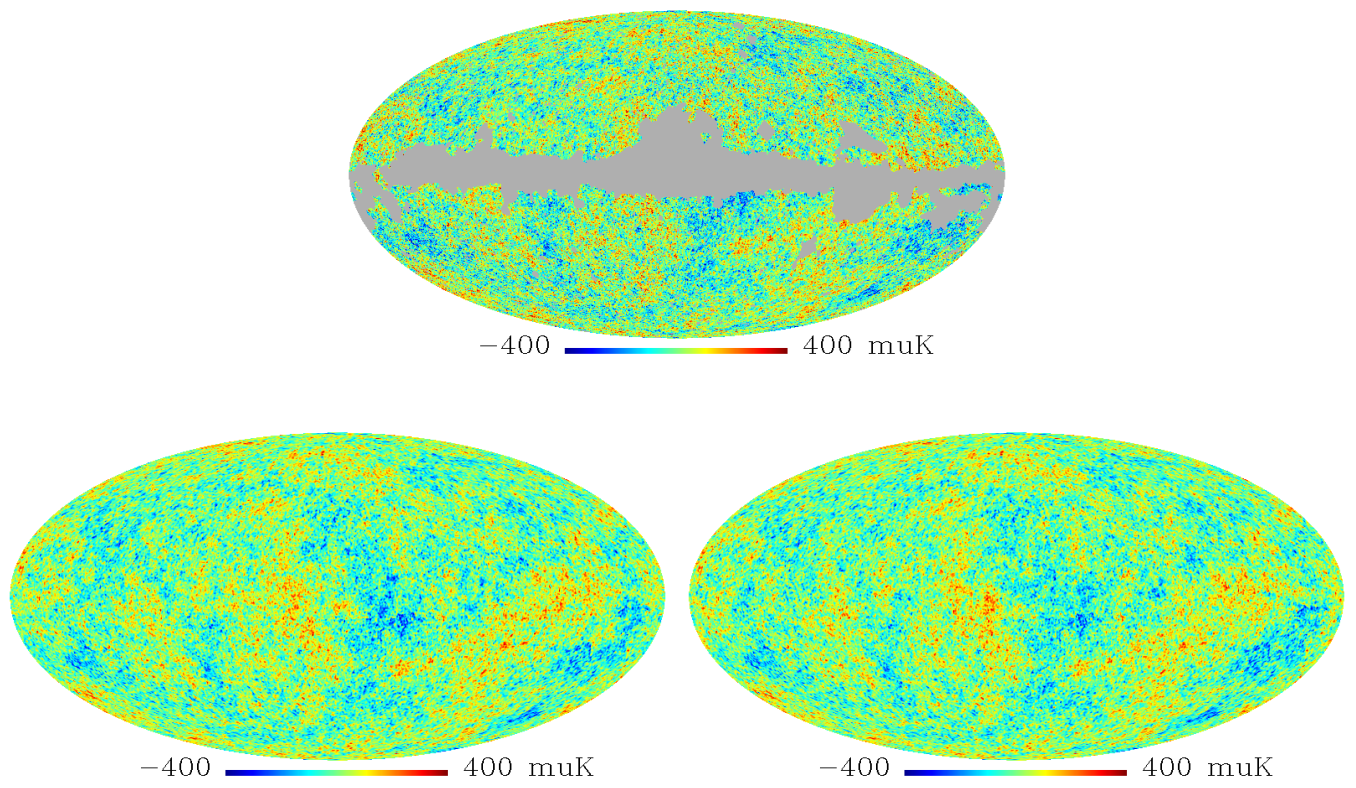

Figure 6: Restored maps from the foreground-reduced map of $\mathrm{W}$ band, two restored maps (middle and bottom)

the noise power spectrum, we used the WMAP noise model $\sigma_{0} / \sqrt{N_{\mathrm{obs}}}$, where $\sigma_{0}$ is $2.197 \mathrm{mK}$, $3.137 \mathrm{mK}$ and $6.549 \mathrm{mK}$ for $\mathrm{Q}, \mathrm{V}$, and $\mathrm{W}$ band respectively, and $N_{\mathrm{obs}}$ is the number of observation for the pixel [ए]]. We generated 1000 simulated noise maps, from which we estimated the noise power spectrum $N_{l}$. In Fig. 团, \ and G, we show the restored whole-sky maps. For each frequency band, we show two restored maps. Each restored maps have the beam smoothing of the original maps, which are all different for each band. Note that all restored maps are generated from different unconstrained realizations for all restoration, restored maps from different bands have different morphology inside the masked area, besides the beam smoothing. From the restored maps, we estimated the quadrupole and octupole components.

Using the quadrupole and octupole anisotropy of restored maps, we investigated the anomalous alignment between the quadrupole and octupole, which are found in the multipole vector analysis of the ILC map [2]-[23]. In the original study by [2]-[23], three dot products $D_{1}, D_{2}$, and $D_{3}$ were estimated, where the most anomalous alignment is associated with $D_{1}$. Since our restoration method is statistical, we generated 10,000 restored maps for $\mathrm{Q}, \mathrm{V}$ and $\mathrm{W}$ bands respectively. From each restored map, we computed the alignment between multipole vectors, which are quantified by three dot products $D_{1}, D_{2}$, and $D_{3}$, where a higher value of a dot product correspond to higher alignment [2]-[23]. For $D_{1}$, we find most of restored maps have even more anomalous alignment than the ILC map. It is also interesting to note that $D_{2}$ and $D_{3}$ values of the $\mathrm{V}$ band map are much higher than the other bands, even though the $\mathrm{V}$ band map is expected to contain least foreground contamination. Given our result, we find it difficult to attribute the anomalous alignment to the residual foregrounds. Previously, [2], 37] investigated anisotropy at lowest multipoles, by applying the power equalization filter to the cut-sky foreground-reduced maps. Our result is consistent with 

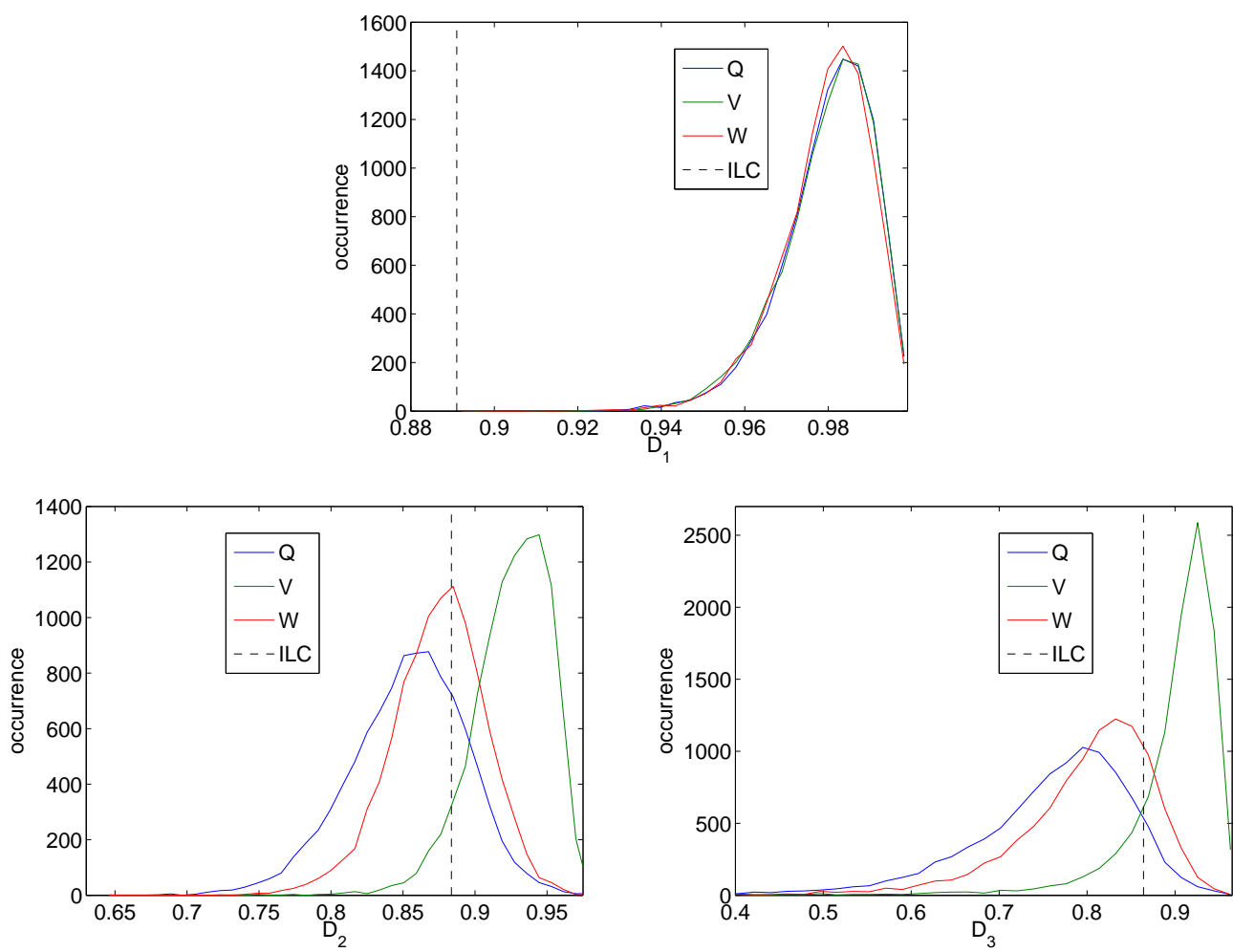

Figure 7: the distribution of the multipole vector alignment between the quadrupole and octupole components of restored maps: the alignment of the whole-sky ILC is marked as dashed lines for comparison.

their finding that the alignment anomaly is robust with respect to the frequency and sky cut [ [य, [3]].

\section{Discussion}

Foreground masking leads to large cuts around the Galactic equator and numerous holes in the CMB map data. Therefore, there have been a lot of effort on restoring the CMB data to a wholesky maps. There have been well-established methods on constrained Monte-Carlo simulation for Gaussian fields. Therefore, we may restore a statistical ensemble of a whole-sky maps, given the data, with the assumption that our early Universe is Gaussian and statistically isotropic. However, we may not readily apply the method, due to the prohibitive computational cost. Noting this, we implemented in-painting in harmonic space, where the computational load may be greatly reduced with good approximation. We validated our method with simulations, and subsequently applied it to the WMAP data. From the simulation result, we found the the restoration is made in a way compliant with the expected statistical properties. Using the restored maps from the masked WMAP foreground-reduced maps, we investigated the anomalous alignment between the quadrupole and octupole components, which are originally found in the multipole vector analysis of the WMAP whole-sky ILC map. From the analysis, we find the alignment in the restored maps is even higher than that of the ILC map. We also notice that $\mathrm{V}$ band maps show rather higher alignment than other bands, despite the expectation of the $\mathrm{V}$ band map being cleanest. Therefore, we find it hard 
to attribute the alignment to residual foregrounds. We believe our method will be of great use for the Planck data analysis and future missions.

\section{Acknowledgments}

We acknowledge the use of the Legacy Archive for Microwave Background Data Analysis (LAMBDA). Our data analysis made the use of HEALPix and SpICE.

\section{References}

[1] G. Efstathiou. A maximum likelihood analysis of the low cosmic microwave background multipoles from the Wilkinson Microwave Anisotropy Probe. Mon. Not. R. Astron. Soc., 348:885-896, March 2004. arXiv:astro-ph/0310207.

[2] P. Bielewicz, K. M. Górski, and A. J. Banday. Low-order multipole maps of cosmic microwave background anisotropy derived from WMAP. Mon. Not. R. Astron. Soc., 355:1283-1302, December 2004. arXiv:astro-ph/0405007.

[3] A. de Oliveira-Costa and M. Tegmark. CMB multipole measurements in the presence of foregrounds. Phys. Rev. D, 74(2):023005, July 2006. arXiv:astro-ph/0603369.

[4] S. M. Feeney, H. V. Peiris, and A. Pontzen. Avoiding bias in reconstructing the largest observable scales from partial-sky data. Phys. Rev. D, 84(10):103002, November 2011. arXiv:1107.5466.

[5] P. Abrial, Y. Moudden, J.-L. Starck, J. Fadili, J. Delabrouille, and M. K. Nguyen. CMB data analysis and sparsity. Statistical Methodology, 5:289-298, July 2008. arXiv:0804.1295.

[6] K. T. Inoue, P. Cabella, and E. Komatsu. Harmonic in-painting of the cosmic microwave background sky: Formulation and error estimate. Phys. Rev. D, 77(12):123539, June 2008. arXiv:0804.0527.

[7] M. Bucher and T. Louis. Filling in CMB map missing data using constrained Gaussian realizations. September 2011. arXiv:1109.0286.

[8] Simon Masnou. Disocclusion: a variational approach using level lines. IEEE Transactions on Image Processing, 11:68-76, 2002.

[9] C Ballester, M. BertalmÃ $\mathbf{m o , ~ V . ~ C a s e l l e s , ~ G . ~ S a p i r o , ~ a n d ~ J ~ V e r d e r a . ~ F i l l i n g - I n ~ b y ~ J o i n t ~ I n t e r p o l a t i o n ~ o f ~}$ Vector Fields and Gray Levels. IEEE Trans. Image Processing, 10:1200 - 1211, 2001.

[10] M. Bertalmio , L. Vese, G. Sapiro, and G. Osher. Simultaneous structure and texture image in-painting. IEEE Trans. Image Processing, 12:882 - 889, 2003.

[11] S. D. Rane, G. Sapiro, and M. and Bertalmio. Structure and texture filling-in of missing image blocks in wireless transmission and compression applications. IEEE Trans. Image Processing, 12:296 -303, 2003.

[12] E. Bertschinger. Path integral methods for primordial density perturbations - Sampling of constrained Gaussian random fields. Astrophys. J. Lett., 323:L103-L106, December 1987.

[13] Y. Hoffman and E. Ribak. Primordial Gaussian perturbation fields - Constrained realizations. Astrophys. J., 384:448-452, January 1992.

[14] Y. Hoffman and E. Ribak. Constrained realizations of Gaussian fields - A simple algorithm. Astrophys. J. Lett., 380:L5-L8, October 1991. 
[15] H. K. Eriksen, I. J. O’Dwyer, J. B. Jewell, B. D. Wandelt, D. L. Larson, K. M. Górski, S. Levin, A. J. Banday, and P. B. Lilje. Power Spectrum Estimation from High-Resolution Maps by Gibbs Sampling. Astrophys. J., 155:227-241, December 2004.

[16] B. D. Wandelt, D. L. Larson, and A. Lakshminarayanan. Global, exact cosmic microwave background data analysis using Gibbs sampling. Phys. Rev. D, 70(8):083511, October 2004. arXiv:astro-ph/0310080.

[17] E. Komatsu, K. M. Smith, J. Dunkley, C. L. Bennett, B. Gold, G. Hinshaw, N. Jarosik, D. Larson, M. R. Nolta, L. Page, D. N. Spergel, M. Halpern, R. S. Hill, A. Kogut, M. Limon, S. S. Meyer, N. Odegard, G. S. Tucker, J. L. Weiland, E. Wollack, and E. L. Wright. Seven-year Wilkinson Microwave Anisotropy Probe (WMAP) Observations: Cosmological Interpretation. Astrophys.J.Suppl., 192:18-+, February 2011. arXiv:1001.4538.

[18] N. Jarosik, C. L. Bennett, J. Dunkley, B. Gold, M. R. Greason, M. Halpern, R. S. Hill, G. Hinshaw, A. Kogut, E. Komatsu, D. Larson, M. Limon, S. S. Meyer, M. R. Nolta, N. Odegard, L. Page, K. M. Smith, D. N. Spergel, G. S. Tucker, J. L. Weiland, E. Wollack, and E. L. Wright. Seven-year Wilkinson Microwave Anisotropy Probe (WMAP) Observations: Sky Maps, Systematic Errors, and Basic Results. Astrophys.J.Suppl., 192:14-+, February 2011. arXiv:1001.4744.

[19] B. Gold, N. Odegard, J. L. Weiland, R. S. Hill, A. Kogut, C. L. Bennett, G. Hinshaw, X. Chen, J. Dunkley, M. Halpern, N. Jarosik, E. Komatsu, D. Larson, M. Limon, S. S. Meyer, M. R. Nolta, L. Page, K. M. Smith, D. N. Spergel, G. S. Tucker, E. Wollack, and E. L. Wright. Seven-year Wilkinson Microwave Anisotropy Probe (WMAP) Observations: Galactic Foreground Emission. Astrophys.J.Suppl., 192:15-+, February 2011. arXiv:1001.4555.

[20] Angélica de Oliveira-Costa, Max Tegmark, Matias Zaldarriaga, and Andrew Hamilton. The significance of the largest scale CMB fluctuations in WMAP. Phys. Rev. D, 69:063516, 2004.

[21] C. J. Copi, D. Huterer, and G. D. Starkman. Multipole vectors: A new representation of the CMB sky and evidence for statistical anisotropy or non-Gaussianity at $2 \leq l \leq 8$. Phys. Rev. D, 70(4):043515-+, August 2004.

[22] D. J. Schwarz, G. D. Starkman, D. Huterer, and C. J. Copi. Is the Low- $\ell$ Microwave Background Cosmic? Physical Review Letters, 93(22):221301-+, November 2004.

[23] C. J. Copi, D. Huterer, D. J. Schwarz, and G. D. Starkman. On the large-angle anomalies of the microwave sky. Mon. Not. R. Astron. Soc., 367:79-102, March 2006.

[24] K. Land and J. Magueijo. Examination of Evidence for a Preferred Axis in the Cosmic Radiation Anisotropy. Physical Review Letters, 95(7):071301-+, August 2005.

[25] K. Land and J. Magueijo. The Axis of Evil revisited. Mon. Not. R. Astron. Soc., 378:153-158, June 2007.

[26] A. Rakić and D. J. Schwarz. Correlating anomalies of the microwave sky. Phys. Rev. D, 75(10):103002-+, May 2007.

[27] K. Land and J. Magueijo. Is the Universe odd? Phys. Rev. D, 72(10):101302-+, 2005.

[28] P. Coles, P. Dineen, J. Earl, and D. Wright. Phase correlations in cosmic microwave background temperature maps. Mon. Not. R. Astron. Soc., 350:989-1004, May 2004. arXiv:astro-ph/0310252.

[29] L.-Y. Chiang, P. D. Naselsky, O. V. Verkhodanov, and M. J. Way. Non-Gaussianity of the Derived Maps from the First-Year Wilkinson Microwave Anisotropy Probe Data. Astrophys. J. Lett., 590:L65-L68, June 2003. 
[30] J. Kim and P. Naselsky. Anomalous Parity Asymmetry of the Wilkinson Microwave Anisotropy Probe Power Spectrum Data at Low Multipoles. Astrophys. J. Lett., 714:L265-L267, May 2010.

[31] J. Kim and P. Naselsky. Anomalous parity asymmetry of WMAP power spectrum data at low multpoles: is it cosmological or systematics? Phys. Rev. D, 82(6), January 2010. arXiv:1002.0148.

[32] C. J. Copi, D. Huterer, D. J. Schwarz, and G. D. Starkman. Large angle anomalies in the CMB. April 2010. arXiv:1004.5602.

[33] C. J. Copi, D. Huterer, D. J. Schwarz, and G. D. Starkman. Bias in low-multipole CMB reconstructions. March 2011. arXiv:1103.3505.

[34] M. Hansen, A. M. Frejsel, J. Kim, P. Naselsky, and F. Nesti. Pearson's random walk in the space of the cmb phases: Evidence for parity asymmetry. Phys. Rev. D, 83(10):103508, May 2011.

[35] P. Naselsky, M. Hansen, and J. Kim. Symmetry of the CMB sky as a new test of its statistical isotropy. Non cosmological octupole? JCAP, 9:12, September 2011. arXiv:1105.4426.

[36] P. Naselsky, W. Zhao, J. Kim, and S. Chen. Is the CMB asymmetry due to the kinematic dipole? Submitted to Astrophys. J., August 2011. arXiv:1108.4376.

[37] P. Bielewicz, H. K. Eriksen, A. J. Banday, K. M. Górski, and P. B. Lilje. Multipole Vector Anomalies in the First-Year WMAP Data: A Cut-Sky Analysis. Astrophys. J., 635:750-760, December 2005. arXiv:astro-ph/0507186. 